\title{
Laparoscopic needle assisted extracorporeal repair of inguinal hernia in children: our experience
}

\begin{abstract}
Purpose: Despite of some controversy, laparoscopic repair of inguinal hernia has emerged recently as an alternative technique for open hernia repair in children. Various methods of laparoscopic repair have been described. Basic principle is to close the internal inguinal ring either by intra corporeal or by extracorporeal suturing. This study was done to evaluate our experience in laparoscopic needle assisted extracorporeal repair of inguinal Hernia in Children.
\end{abstract}

Methods: This retrospective study was done in a tertiary Pediatric Surgical center at Dhaka, Bangladesh from January 2012 to June 2017. Medical records of the cases who underwent inguinal hernia repaired laparoscopicaly with needle assisted extracorporeal knot were reviewed. The variables to evaluate the patients included: demographics, surgical time, complications, recurrence and follow up time. The cases with incomplete data were excluded.

Result: Total 202 hernias of 131 patients underwent laparoscopic assisted extracorporeal inguinal hernia repairs. Age of the patients at operation was ranged from lyear 3 months to 7 years 8 months. Preoperative diagnosed unilateral hernias were 112 cases. Twenty six (19.9\%) patients found per-operative contra lateral patent procesus virginals. Mean operative time was 21.2 minutes for unilateral and 30.3 minutes for bilateral repair. Six patients developed mild bleeding from needle puncture site, 5 boys developed transient hydroceles and four $(1.98 \%)$ cases developed recurrences hernia. Mean follow up time was 16.3 months.

Conclusion: It can be conclude that Laparoscopic Needle Assisted Extracorporeal repair of Inguinal Hernia is simple, safe, and efficacious for pediatric patients. It can be performed by the surgeons who are in the initial stage of learning laparoscopic surgery.

Keywords: Pediatric Inguinal Hernia, Laparoscopic Hernia Repair, Extra corporeal knot
Volume 8 Issue 2 - 2018

\author{
Kazi Md Noor-ul Ferdous,' Md Samiul \\ Hasan, ${ }^{2}$ Md Delwar Hossain, ${ }^{3}$ Md \\ Muhammad Rashedul Alam, ${ }^{4}$ Md \\ Abdus Sattar, ${ }^{5}$ M Kabirul Islam ${ }^{6}$ \\ 'Department of Neonatal Surgery; Division of Pediatric \\ Surgery, Bangladesh Institute of Child Health and Dhaka Shishu \\ (Children) Hospital, Bangladesh \\ ${ }^{2}$ Department of Minimally Invasive Surgery, Division of Pediatric \\ Surgery Bangladesh Institute of Child Health and Dhaka Shishu \\ (Children) Hospital, Bangladesh \\ ${ }^{3}$ Department of Pediatric Neurosurgery, Division of Pediatric \\ Surgery Bangladesh Institute of Child Health and Dhaka Shishu \\ (Children) Hospital, Bangladesh \\ ${ }^{4}$ Department of Pediatric Burn \& Reconstructive Surgery, \\ Division of Pediatric Surgery, Bangladesh Institute of Child \\ Health and Dhaka Shishu (Children) Hospital, Bangladesh \\ ${ }^{5}$ Department of Pediatric Surgery, Pabna Mesiacal College \& \\ Hospital, Pabna, Bangladesh \\ ${ }^{6}$ Department of Neonatal Surgery, Division of Pediatric \\ Surgery, Bangladesh Institute of Child Health and Dhaka Shishu \\ (Children) Hospital, Bangladesh
}

Correspondence: Kazi Md Noor-ul Ferdous, Hasan, MBBS, MS, Department of Neonatal Surgery, Division of Pediatric Surgery, Bangladesh Institute of Child Health and Dhaka Shishu (Children) Hospital Dhaka Shishu, (Children) Hospital Dhaka, Bangladesh,Email kmnferdous@gmail.com

Received: January 28, 2018 | Published: March21, 2018

\section{Introduction}

One of the most frequently performed operative procedures in pediatric age group is inguinal herniotomy. The traditional surgical approach is an exploration of the inguinal canal, dissection of the hernia sac from the spermatic cord and the hernia sac is closed at the level of the internal inguinal ring. ${ }^{1}$ To evaluate contra lateral defects during open inguinal hernia repair, laparoscopic telescope has been used for long time in paediatric age group. ${ }^{2}$ Since the first time of laparoscopic inguinal hernia repaired by El-Gohary, it has been performed in many institutions with a variety of methods. ${ }^{2,3}$ Although, laparoscopic inguinal hernia repair in children is getting popularity as a safe \& feasible method, laparoscopic inguinal hernia repair in children is still a controversial topic. ${ }^{4,5}$ Usually the laparoscopic procedure is performed through three or, rarely, two ports in the abdominal wall and requires intra peritoneal suturing ${ }^{6,7}$ which is one of the most difficult technical aspects of laparoscopy. Even with adequate experience, Intra-abdominal suturing involves many manipulations with instruments inside the abdominal cavity and carries the risk of visceral injury. ${ }^{1}$ Recently, many authors suggested that laparoscopic hernia repair with extracorporeal ligation, as compared with intracorporeal suturing, results marked reduction in operative time, low recurrence rates, and excellent cosmetic results. ${ }^{8}$ In extracorporeal technique, the suture is placed through the puncture-point in the skin with an injection needle of various sizes and tied around the hernia sac at the level of the inguinal ring. The technique requires only umbilical port for telescope and some authors used a Maryland Forceps, introduced directly without any port, which leaves almost invisible scar. ${ }^{1,2,8,9}$ In our centre, we repair the defect in the internal inguinal ring using laparoscopic visualization and extracorporeal knot tying. The goal of this study is to evaluate our experiences of the laparoscopic needle assisted extracorporeal repair of inguinal Hernia in Children.

\section{Materials and methods}

This retrospective study was done in the Division of Pediatric Surgery; Bangladesh Institute of Child Health (BICH) \& Dhaka Shishu (Children) Hospital, Dhaka, Bangladesh during the past 5 years 6 months (January 2012 to July 2017). We reviewed the medical records of the cases that underwent inguinal hernia repaired laparoscopicaly with needle assisted extracorporeal knot. The variables to evaluate the patients included: demographics, surgical time (from the incision to last stitch), complications, recurrence and follow up time. We 
excluded the cases with incomplete data. Permission from the Ethical committee of the institute was taken. Statistical significance is determined by using the SPSS software, version 22. Numerical data were expressed in mean $\pm \mathrm{SD}$ and categorical data were expressed as percentage.

\section{Procedure}

The procedures were done under general endotracheal anaesthesia. Pneumoperitonium was made by closed technique through the infraumbilicus wound and a 5-mm trocar was introduced. A 5-mm 300 scope trocar used to visualize the internal inguinal ring and confirm the presence of hernia. The contra lateral internal ring is also inspected for a patent procesus vaginalis. In the absence of clinical symptoms, we repaired these defects as potential hernias. Through lateral border of the rectus abdominis either at the level or just below the umbilicus, a 3-mm Maryland forceps was inserted directly. Site of the internal ring closure was identified by finger pressure on the abdominal wall at the site of internal ring. A blood transfusion needle (18-gauge) threaded with a 3-0 prolene in the form of a loop was inserted through the skin and pushed down along one side of the internal inguinal ring just beneath the peritoneum.

The peritoneum was lifted with Maryland forceps to make it easy passage of the needle and also to protect the vas deferens and spermatic vessels or the round ligament. Peritoneum was punctured by the needle just lateral to the vas and vessels. The needle was withdrawn after holding the apex of the prolene loop with the Maryland. The needle was then threaded just short of its tip with long end of same prolene ${ }^{\circledR}$ outside the abdomen and reinserted through the same needle punctured wound along other side of the ring in similarly. The peritoneum was punctured again just medial to the vas and vessels. Then the prolene thread was pushed through the needle for sufficient length, was held by the Maryland forceps and the needle was withdrawn. Free end of the thread was then passed through the intra-peritoneal prolene loop sufficiently. The prolene loop was then withdrawn from outside which kinked the long free end of prolene thread and pulled it out. Thus the prolene thread made a loop around the internal ring, which was then tied extra-corporeally to close the internal ring. Any gas trapped inside the scrotum or labia should be squeezed out before closing the ring. A second purse string may require to close a very large defect. The umbilical port wound and Maryland wound were closed. All the patients were discharged on the next day and called for follow- up visits at 2 weeks, 1 month, 6 months, and 1 year. During follow-up, outcome measurements- scrotal edema, postoperative hydroceles formation, iatrogenic testicular ascent, testicular atrophy, recurrence rate, and cosmetic results were evaluated.

\section{Results}

Total 131 patients underwent laparoscopic assisted extra-corporeal inguinal hernia repairs during the 66 months of study period. Mean age of the patients at operation was 48.2 months, ranging from 1year 3 months to 7 year 8 months. Preoperative diagnosed right-sided hernias were repaired in 60 , left in 52, and bilateral in 19 cases. Twenty six (19.9\%) patients found per-operative contra lateral patent processes vaginalis and air insufflations demonstrated a hernia in the groin or labia. All these were repaired at the same sitting. Thus a total of 202 hernias repaired in this study. Mean operative time was 21.2 minutes (Range: 14-25 minutes) for single-sided repair and 30.3 minutes (Range: 20-38 minutes) for bilateral repair. During operation, no patients developed injuries to the vas deferens and no episodes of major bleeding were identified. Minor complications included, 6 patients developed mild bleeding from needle puncture site, which resolved on pressure. On follow up, we found four (1.98\%) case of recurrent hernia in three boys within 2 to 4 months postoperatively. Transient hydroceles were observed in five boys and that disappeared spontaneously after 3 to 5 months. No other major complications like testicular atrophy, testicular ascent was observed. Follow up time since surgery was range from 3-36 months (Mean 16.3 months).

\section{Discussion}

Though our centre is the largest paediatric surgical centre in the country, we perform very limited laparoscopic surgery. Due to work overload, limited resource and long learning curve we are still at the beginning of laparoscopic surgery. Due to limited experience we did not perform laparoscopic hernia repair below 1 year of age, recurrent hernia, hernia in obese patients, obstructed and incarcerated hernia and patients with congenital heart disease The advantage of laparoscopic inguinal hernia repair in children is that the direct approach to the internal inguinal ring avoids opening the inguinal canal, easy evaluation of the contra lateral side, minimum dissection and avoidance of injury to vas and vessels and iatrogenic ascent of the testis, and decreased operating time and reduced incidence of testicular atrophy. ${ }^{1,4,8,10-13}$ In this study, we have close the defect in the internal inguinal ring using 5-mm umbilical port for laparoscopic visualization, a Maryland forceps introduced directly through a small stub wound lateral to the rectus muscle and an $18 \mathrm{G}$ or $20 \mathrm{G}$ needle (depending on the ages) treated with $3 / 0$ polypropylene within it inserted at the midpoint of the inguinal line for extra-corporeal knot tying.

In our series, contra lateral hernias were detected in $19.84 \%$ cases and were repaired at the same time. This result is similar to most reported series. Evaluation of contra lateral processes vaginalis is one of the main favouring point for laparoscopic Hernia repair. ${ }^{1,2,5,9,11}$ Literatures shows mean operative time for laparoscopic needle assisted extracorporeal unilateral repair was 10.5-20.5 minutes and in bilateral it was 20-26.4 minutes. ${ }^{1,2,9,11,12}$ In our study, the mean operative times were a bit longer; it was due to long learning period. Manoz Shaha felt the same problem in his series. ${ }^{12}$ In our series, complications like mild bleeding from needle puncture site, transient hydroceles and recurrence noted in few cases. Use of Trend Ellensburg's position and needle puncture under direct camera vision can avoid mostly feared gut and vessel injury. ${ }^{11}$ The studies those reviewed recurrence in open and laparoscopic hernia repairs shown, the average time to recurrence in open was between 1 to 2 years and in laparoscopy, recurrences seem to occur earlier, often within the first 6 months. ${ }^{13-15}$ Open herniotomy in children has been reported to have recurrence rates of $0.8-3.8 \%$, while in laparoscopic hernia repair, it ranged from 0.7 to $4.5 \% .^{12}$

The recurrence rate is higher for the laparoscopic treatment of inguinal hernia than the traditional open approach, although the difference is not significant [1]. Complete encirclement of the ring, emptying the sac, and reducing the abdominal pressure before tying may help in reducing hydroceles formation and recurrence of hernia [12]. On follow up, we found four case of recurrences hernia within 4 months which were repaired by open technique after 6 months. Transient hydroceles were observed in five boys and that disappeared spontaneously after 3 to 5 months. Pat kowski et al. ${ }^{1}$ described in their study, intra corporeal suturing is one of the most difficult technical aspects of laparoscopy as it requires many manipulations with instruments inside the abdominal cavity and carries the risk of visceral injury. ${ }^{1}$ In extracorporeal technique, we found the suture placement is very safe and easy and many authors supported this finding. ${ }^{1,2,8,9}$ So we recommend this technique specially for surgeons in their initial experience in laparoscopy. 


\section{Conclusion}

It can be concluded that Laparoscopic Needle Assisted Extracorporeal repair of inguinal Hernia is simple, safe, and efficacious for paediatric patients. It does not need any additional working port with excellent cosmetic results and low complications. It can be performed by the surgeons who are in the initial stage of learning laparoscopic surgery.

\section{Acknowledgment}

None.

\section{Conflict of interest}

None.

\section{References}

1. Patkowski D, Czernik J, Chrzan R, et al. Percutaneous Internal Ring Suturing: A Simple Minimally Invasive Technique for Inguinal Hernia Repair in Children. J Laparoendosc Adv Surg Tech A. 2006;16(5):513417.

2. Yukihiro Tatekawa. Laparoscopic Extracorporeal Ligation of Hernia Defects Using an Epidural Needle and Preperitoneal Hydrodissection. $J$ Endourol. 2012;26(5):474-477.

3. El-Gohary MA. Laparoscopic ligation of inguinal hernia in girls. Pediatric Endosurgery \& Innovative Techniques. 1997;1(3):185-188.

4. Lukong CS. Surgical techniques of laparoscopic inguinal hernia repair in childhood: a critical appraisal. J Surg Tech Case Rep. 2012;4:1-5.

5. Ozgediz D, Roayaie K, Lee H, et al. Subcutaneous endoscopically assisted ligation (SEAL) of the internal ring for repair of inguinal hernias in children: report of a new technique and early results. Surg Endosc. 2007;21:1327-1331.
6. Gorsler CM, Schier F. Laparoscopic herniorrhaphy in children. Surg Endosc.2003;17(4):571-573.

7. Li L, Jun Z, Jingbo F, et al. Intra corporeal single-hand knot techniques. Pediatr Endosurg Innovat Techn. 2004;8:1-2.

8. Shalaby R, Ismail M, Dorgham A, et al. Laparoscopic hernia repair in infancy and childhood: Evaluation of two different techniques. $J$ Pediatr Surg. 2010; 45(11):2210-2216.

9. McClain L, Streck C, Lesher A, et al. Laparoscopic needle-assisted inguinal hernia repair in 495 children. Surg Endosc. 2015 ;29(4):7817816.

10. Chan KL, Hui WC, Tam PK. Prospective randomized single-center, single-blind comparison of laparoscopic versus open repair of pediatric inguinal hernia. Surgical Endoscopy And Other Interventional Techniques. 2005;19(7):927-932.

11. Bharathi RS, Dabas AK, Arora M, et al. Laparo- scopic ligation of internal ring-three ports versus single-port technique: are working ports necessary?. J Laparoendosc Adv Surg Tech A. 2008;18(6):891-894.

12. Manoj Saha. Laparoscopic Extracorporeal Ligation of the Internal Inguinal Ring by a Spinal Needle: a Simple Method of Hernia Repair in Children. Indian Journal of Surgery. 2016;78(2):85-89.

13. Ein SH, Njere I, Ein A. Six thousand three hundred sixty- one pediatric inguinal hernias: a 35-year review. J Pediatr Surg. 2006 41(5):980-986.

14. Grosfeld JL, Minnick K, Shedd F, et al. Inguinal hernia in children: factors affecting recur- rence in 62 cases. J Pediatr Surg. 1991;26(3):283-287.

15. Treef W, Schier F. Characteristics of laparoscopic inguinal hernia recurrences. Pediatr Surg Int. 2009;25(2):149-152. 